\title{
625.
}

\section{ON THE CONDITION FOR THE EXISTENCE OF A SURFACE CUTTING AT RIGHT ANGLES A GIVEN SET OF LINES.}

[From the Proceedings of the London Mathematical Society, vol. viII. (1876-1877), pp. 53-57. Read December 14, 1876.]

In a congruency or doubly infinite system of right lines, the direction-cosines. $\alpha, \beta, \gamma$ of the line through any given point $(x, y, z)$, are expressible as functions of $x, y, z$; and it was shown by Sir W. R. Hamilton in a very elegant manner that, in order to the existence of a surface (or, what is the same thing, a set of parallel surfaces) cutting the lines at right angles, $\alpha d x+\beta a a^{7} y+\gamma d z$ must be an exact differential: when this is so, writing $V=\int(\alpha d x+\beta d y+\gamma d z)$, we have $V=c$, the equation of the system of parallel surfaces each cutting the given lines at right angles.

The proof is as follows:-If the surface exists, its differential equation is $\alpha d x+\beta d y+\gamma d z=0$, and this equation must therefore be integrable by a factor. Now the functions $\alpha, \beta, \gamma$ are such that $\alpha^{2}+\beta^{2}+\gamma^{2}=1$, and they besides satisfy a system of partial differential equations which Hamilton deduces from the geometrical notion of a congruency; viz. passing from the point $(x, y, z)$ to the consecutive point on the line, that is, to the point whose coordinates are $x+\rho \alpha, y+\rho \beta, z+\rho \gamma$ ( $\rho$ infinitesimal), the line belonging to this point is the original line; and consequently $\alpha, \beta, \gamma$, considered as functions of $x, y, z$, must remain unaltered when these variables are changed into $x+\rho \alpha, y+\rho \beta, z+\rho \gamma$, respectively. We thus obtain the equations

$$
\begin{aligned}
& \alpha \frac{d \alpha}{d x}+\beta \frac{d x}{d y}+\gamma \frac{d \alpha}{d z}=0 \\
& \alpha \frac{d \beta}{d x}+\beta \frac{d \beta}{d y}+\gamma \frac{d \beta}{d z}=0 \\
& \alpha \frac{d \gamma}{d x}+\beta \frac{d \gamma}{d y}+\gamma \frac{d \gamma}{d z}=0
\end{aligned}
$$


Combining herewith the equations obtained by differentiation of $\alpha^{2}+\beta^{2}+\gamma^{2}=1$, viz.

$$
\begin{aligned}
& \alpha \frac{d \alpha}{d x}+\beta \frac{d \beta}{d x}+\gamma \frac{d \gamma}{d x}=0 \\
& \alpha \frac{d \alpha}{d y}+\beta \frac{d \beta}{d y}+\gamma \frac{d \gamma}{d y}=0 \\
& \alpha \frac{d \alpha}{d z}+\beta \frac{d \beta}{d z}+\gamma \frac{d \gamma}{d z}=0
\end{aligned}
$$

and subtracting the corresponding equations, we obtain three equations which may be written

or, what is the same thing,

$$
\alpha: \beta: \gamma=\frac{d \beta}{d z}-\frac{d \gamma}{d y}: \frac{d \gamma}{d x}-\frac{d \alpha}{d z}: \frac{d \alpha}{d y}-\frac{d \beta}{d x}
$$

$$
\frac{d \beta}{d z}-\frac{d \gamma}{d y}, \frac{d \gamma}{d x}-\frac{d \alpha}{d z}, \frac{d \alpha}{d y}-\frac{d \beta}{d x}=k \alpha, k \beta, k \gamma
$$

and, multiplying by $\alpha, \beta, \gamma$, and adding,

$$
k=\alpha\left(\frac{d \beta}{d z}-\frac{d \gamma}{d y}\right)+\beta\left(\frac{d \gamma}{d x}-\frac{d \alpha}{d z}\right)+\gamma\left(\frac{d \alpha}{d y}-\frac{d \beta}{d x}\right) .
$$

We thus see that, if the function on the right-hand vanish, then $k=0$, and consequently also

$$
\frac{d \beta}{d z}-\frac{d \gamma}{d y}, \frac{d \gamma}{d x}-\frac{d \alpha}{d z}, \frac{d \alpha}{d y}-\frac{d \beta}{d x} \text { each }=0
$$

viz. if the equation $\alpha d x+\beta d y+\gamma d z=0$ be integrable, then $\alpha d x+\beta d y+\gamma d z$ is an exact differential; which is the theorem in question.

But it is interesting to obtain the first mentioned set of differential equations from the analytical equations of a congruency, viz. these are $x=m z+p, y=n z+q$, where $m, n, p, q$ are functions of two arbitrary parameters, or, what is the same thing, $p, q$ are given functions of $m, n$; and therefore, from the three equations, $m, n$ are given functions of $x, y, z$. And it is also interesting to express in terms of these quantities $m, n$, considered as functions of $x, y, z$, the condition for the existence of the set of surfaces.

We have

$$
\alpha, \beta, \gamma=\frac{m}{R}, \frac{n}{R}, \frac{1}{R}, \text { where } R=\sqrt{1+m^{2}+n^{2}} ;
$$

and thence without difficulty

$$
\begin{aligned}
& \left(\alpha \frac{d}{d x}+\beta \frac{d}{d y}+\gamma \frac{d}{d z}\right) \alpha=\frac{1}{R^{4}}\left[\left(1+n^{2}\right)\left(m \frac{d m}{d x}+n \frac{d m}{d y}+\frac{d m}{d z}\right)-m n\left(m \frac{d n}{d x}+n \frac{d n}{d y}+\frac{d n}{d z}\right)\right] \\
& \left(\alpha \frac{d}{d x}+\beta \frac{d}{d y}+\gamma \frac{d}{d z}\right) \beta=\frac{1}{R^{4}}\left[-m n(\quad)+\left(1+m^{2}\right)\left(\begin{array}{l}
\text { " } \\
\left(\alpha \frac{d}{d x}+\beta \frac{d}{d y}+\gamma \frac{d}{d z}\right) \gamma=\frac{1}{R^{4}}[-(\quad)
\end{array}\right)\right]
\end{aligned}
$$


so that the required equations in $\alpha, \beta, \gamma$ will be satisfied if only

$$
\begin{aligned}
& m \frac{d m}{d x}+n \frac{d m}{d y}+\frac{d m}{d z}=0, \\
& m \frac{d n}{d x}+n \frac{d n}{d y}+\frac{d n}{d z}=0
\end{aligned}
$$

and it is to be shown that these equations hold good. give

Writing for shortness $d p=A d m+B d n, d q=C d m+D d n$, the equations of the line

$$
\begin{array}{rrr}
1=z \frac{d m}{d x}+A \frac{d m}{d x}+B \frac{d n}{d x}, & 0=z \frac{d n}{d x}+C \frac{d m}{d x}+D \frac{d n}{d x}, \\
0=z \frac{d m}{d y}+A \frac{d m}{d y}+B \frac{d n}{d y}, & 1=z \frac{d n}{d y}+C \frac{d m}{d y}+D \frac{d n}{d y}, \\
-m=z \frac{d m}{d z}+A \frac{d m}{d z}+B \frac{d n}{d z}, & -n=z \frac{d n}{d z}+C \frac{d m}{d z}+D \frac{d n}{d z}
\end{array}
$$

or, writing

so that identically

$$
\lambda, \mu, \nu=\frac{d m}{d y} \frac{d n}{d z}-\frac{d m}{d z} \frac{d n}{d y}, \frac{d m}{d z} \frac{d n}{d x}-\frac{d m}{d x} \frac{d n}{d z}, \frac{d m}{d x} \frac{d n}{d y}-\frac{d m}{d y} \frac{d n}{d x},
$$

$$
\begin{aligned}
& \lambda \frac{d m}{d x}+\mu \frac{d m}{d y}+\nu \frac{d m}{d z}=0 \\
& \lambda \frac{d n}{d x}+\mu \frac{d n}{d y}+\nu \frac{d n}{d z}=0
\end{aligned}
$$

then in each set, multiplying by $\lambda, \mu, \nu$ and adding, so as to eliminate $A, B, C, D$, we find

$$
\lambda-m \nu=0, \quad \mu-n \nu=0 .
$$

Substituting these values of $\lambda, \mu$ in the last preceding equations, $\nu$ divides out, and we have the two equations in question.

The foregoing equations give further

$$
A, B, C, D=-z+\frac{1}{\nu} \frac{d n}{d y}, \quad-\frac{1}{\nu} \frac{d m}{d y}, \quad-\frac{1}{\nu} \frac{d n}{d x}, \quad-z+\frac{1}{\nu} \frac{d m}{d x} .
$$

Taking for $\alpha, \beta, \gamma$ the before-mentioned values, we find

$$
\begin{aligned}
\frac{d \alpha}{d y}-\frac{d \beta}{d x} & =\frac{1}{R}\left(\frac{d m}{d y}-\frac{d n}{d x}\right)-\frac{m}{R^{3}}\left(m \frac{d m}{d y}+n \frac{d n}{d y}\right)-\frac{n}{R^{3}}\left(m \frac{d m}{d x}+n \frac{d n}{d x}\right) \\
& =\frac{1}{R^{3}}\left\{\left(1+n^{2}\right) \frac{d m}{d y}-\left(1+m^{2}\right) \frac{d n}{d x}+m n\left(\frac{d m}{d x}-\frac{d n}{d y}\right)\right\}
\end{aligned}
$$

and similarly, but using the equations

$$
m \frac{d m}{d x}+n \frac{d m}{d y}+\frac{d m}{d z}=0, \quad m \frac{d n}{d x}+n \frac{d n}{d y}+\frac{d n}{d z}=0
$$


to eliminate the coefficients $\frac{d m}{d z}, \frac{d n}{d z}$ which in the first instance present themselves, we find

$$
\begin{aligned}
& \frac{d \beta}{d z}-\frac{d \gamma}{d y}=\frac{m}{R^{3}}\left\{\left(1+n^{2}\right) \frac{d m}{d y}-\left(1+m^{2}\right) \frac{d n}{d x}+m n\left(\frac{d m}{d x}-\frac{d n}{d y}\right)\right\}, \\
& \frac{d \gamma}{d x}-\frac{d \alpha}{d z}=\frac{n}{R^{3}}\{\quad ", \quad ",
\end{aligned}
$$

whence, multiplying by $\gamma, \alpha, \beta$, and adding,

$$
\begin{aligned}
\alpha\left(\frac{d \beta}{d z}-\frac{d \gamma}{d y}\right) & +\beta\left(\frac{d \gamma}{d x}-\frac{d \alpha}{d z}\right)+\gamma\left(\frac{d \alpha}{d y}-\frac{d \beta}{d x}\right) \\
& =\frac{1}{1+m^{2}+n^{2}}\left\{\left(1+n^{2}\right) \frac{d m}{d y}-\left(1+m^{2}\right) \frac{d n}{d x}+m n\left(\frac{d m}{d x}-\frac{d n}{d y}\right)\right\}
\end{aligned}
$$

or we have

$$
\left(1+n^{2}\right) \frac{d m}{d y}-\left(1+m^{2}\right) \frac{d n}{d x}+m n\left(\frac{d m}{d x}-\frac{d n}{d y}\right)=0
$$

as the condition for the existence of the set of surfaces.

It is clear that the condition is satisfied when the lines are the normals of a given surface: seeking the surfaces which cut the lines at right angles, we obtain the parallel surfaces; and we are led to the theorem that any parallel surface is the locus of the extremity of a line of constant length measured off from each point of the surface along the normal-or, what is equivalent thereto, the parallel surface is the envelope of a sphere of constant radius having its centre on the surface. I will verify the theorem for the case of the ellipsoid. Taking $X, Y, Z$ as the coordinates of a point on the ellipsoid $\frac{X^{2}}{a^{2}}+\frac{Y^{2}}{b^{2}}+\frac{Z^{2}}{c^{2}}=1$, and $x, y, z$ as current coordinates, the equations of the normal are

$$
\frac{a^{2}}{X}(x-X)=\frac{b^{2}}{Y}(y-Y)=\frac{c^{2}}{Z}(z-Z),(=\lambda \text { suppose }) .
$$

We have therefore

$$
X, Y, Z=\frac{a^{2} x}{a^{2}+\lambda}, \frac{b^{2} y}{b^{2}+\lambda}, \frac{c^{2} z}{c^{2}+\lambda},
$$

and thence

$$
\frac{a^{2} x^{2}}{\left(a^{2}+\lambda\right)^{2}}+\frac{b^{2} y^{2}}{\left(b^{2}+\lambda\right)^{2}}+\frac{c^{2} z^{2}}{\left(c^{2}+\lambda\right)^{2}}=1
$$

an equation which determines $\lambda$ as a function of $x, y, z$.

The direction-cosines $\alpha, \beta, \gamma$ of the normal are proportional to $\frac{X}{a^{2}}, \frac{Y}{b^{2}}, \frac{Z}{c^{2}}$, that 
is, to $\frac{x}{a^{2}+\lambda}, \frac{y}{b^{2}+\lambda}, \frac{z}{c^{2}+\lambda}$, and the equation $\alpha^{2}+\beta^{2}+\gamma^{2}=1$ then determines their absolute magnitudes: the equation $\alpha d x+\beta d y+\gamma d z=d V$ thus is

$$
\frac{\frac{x d x}{a^{2}+\lambda}+\frac{y d y}{b^{2}+\lambda}+\frac{z d z}{c^{2}+\lambda}}{\sqrt{\frac{x^{2}}{\left(a^{2}+\lambda\right)^{2}}+\frac{y^{2}}{\left(b^{2}+\lambda\right)^{2}}+\frac{z^{2}}{\left(c^{2}+\lambda\right)^{2}}}} d V
$$

viz. the left-hand side, considering therein $\lambda$ as a given function of $V$, is an exact differential. We verify this by finding the value of $V$, viz. writing down the two equations

$$
\begin{gathered}
\frac{x^{2}}{\left(a^{2}+\lambda\right)^{2}}+\frac{y^{2}}{\left(b^{2}+\lambda\right)^{2}}+\frac{z^{2}}{\left(c^{2}+\lambda\right)^{2}}-\frac{V^{2}}{\lambda^{2}}=0, \\
\frac{x^{2}}{a^{2}+\lambda}+\frac{y^{2}}{b^{2}+\lambda}+\frac{z^{2}}{c^{2}+\lambda}-\frac{V^{2}}{\lambda}=1,
\end{gathered}
$$

these are equivalent in virtue of the equation that determines $\lambda$; and it is to be shown that, regarding $V$ as given by either of them, say by the second equation, we have for $d V$ its foregoing value. In fact, differentiating the second equation, the term in $d \lambda$ disappears by virtue of the first equation, and the result is

$$
\frac{x d x}{a^{2}+\lambda}+\frac{y d y}{b^{2}+\lambda}+\frac{z d z}{c^{2}+\lambda}-\frac{V d V}{\lambda}=0
$$

in which substituting for $\frac{V}{\lambda}$ its value from the first equation, we have for $d V$ the value in question. Regarding $V$ as a given constant, the two equations give, by elimination of $\lambda$, an equation $\phi(x, y, z, V)=0$, which is, in fact, the surface parallel to the ellipsoid and at a constant normal distance $=V$ from it. 